\title{
GENTES IGUAL QUE TÚ Y QUE YO: LOS TEXTOS DE ROSARIO CASTELLANOS EN LOS CONTRASENTIDOS IDEOLÓGICOS DE LA POLÍTICA LINGÜÍSTICA INDIGENISTA ${ }^{\alpha}$
}

\author{
People like you and me: the texts of Rosario Castellanos \\ in the ideological contradictions of indigenist language policy
}

\section{Susana Ayala Reyes ${ }^{\circledR}$}

Fecha de recepción: 15/09/2020 • Fecha de aceptación: 11/03/2021

Resumen. En este artículo analizo documentos escritos por Rosario Castellanos durante los años que trabajó como guionista de las obras de títeres en los Altos de Chiapas. Estas obras de teatro fueron parte de las campañas educativas dirigidas a la población maya tsotsil y tseltal. Muestro que los discursos circulantes durante los años en los que se construyó la política indigenista estuvieron atravesados por tensiones ideológicas opuestas y contradictorias que se implicaron en la propuesta y uso de categorías de identificación de la población y sus lenguas, los objetivos e ideales teóricos y las historias personales y colectivas de los participantes.

Palabras clave: ideologías lingüísticas; educación indígena; lengua indígena; dialogismo; Rosario Castellanos.

Abstract. In this article we analyze documents written by Rosario Castellanos during the years that she worked as a scriptwriter for the puppet plays in the Highlands of Chiapas. These plays were part of educational campaigns aimed at the Tsotsil and Tseltal Maya population. We show that the discourses circulating during the years in which indigenous politics was being constructed were permeated by opposing and contradictory ideological tensions, tensions

\footnotetext{
${ }^{\alpha}$ Este artículo fue producto de mi estancia en el Programa de Becas Posdoctorales en la UNAM. Centro de Investigaciones Interdisciplinarias en Ciencias y Humanidades, asesorada por la Dra. Paula López Caballero.

B Centro de Investigaciones Interdisciplinarias en Ciencias y Humanidades. UNAM. Torre II de Humanidades, $4 .^{\circ}, 5 .^{\circ}$ y $6 .^{\circ}$ pisos, Circuito Interior, Ciudad Universitaria, Alcaldía Coyoacán, 04510, Ciudad de México, sasybeu@gmail.com (D) https://orcid.org/0000-0003-2676-0254
}

Cómo citar este artículo: Ayala Reyes, Susana. «Gentes igual que tú y que yo: los textos de Rosario Castellanos en los contrasentidos ideológicos de la política lingüística indigenista». Historia y Memoria de la Educación 14 (2021): 391-424 
that were reflected in the proposal and use of categories of identification of the population and their languages, in the theoretical objectives and ideals and in the participants' personal and collective stories.

Keywords: linguistic ideologies; indigenous education; indigenous language; dialogism; Rosario Castellanos.

\section{INTRODUCCIÓN}

En 1951 el Instituto Nacional Indigenista (INI) creó el Centro Coordinador Tzeltal-Tzotzil ${ }^{1}$ (CCI) en los Altos de Chiapas al sureste de México. Esta dependencia instrumentó políticas sociales dirigidas a la población maya tsotsil y tseltal con el fin de encaminar su adscripción como ciudadanos nacionales, la modernización de sus condiciones de vida y su desarrollo económico.

A finales de 1953 los antropólogos que dirigían el CCI impulsaron la formación de titiriteros tsotsiles y tseltales como un entretenimiento que terminó por convertirse en el instrumento principal de las campañas educativas. En 1955, la escritora mexicana Rosario Castellanos se incorporó al proyecto escribiendo guiones para las obras de títeres y trabajar con los titiriteros, instruyéndolos en temas de historia, español y civismo.

Los especialistas que dirigían el CCI consideraban que la ciencia y el arte debían articularse con las acciones políticas. De acuerdo con el historiador Stephen Lewis, ${ }^{2}$ su objetivo era transformar la realidad social que desfavorecía a la población considerada indígena. Si bien la institución no tenía un proyecto oficializado de planificación lingüística, los antropólogos y lingüistas impulsaron tres cambios en los usos del lenguaje dentro de las esferas de la vida cotidiana, la política y la educación.

\footnotetext{
1 En el nombre oficial del Centro Coordinador se usó la ' $z$ ' para tzotzil y tzeltal porque era la forma ortográfica aceptada cuando se creó esa institución. Actualmente, las normas de escritura propuestas por el Instituto Nacional para las Lenguas Indígenas (2011) señalan que la escritura es tsotsil y tseltal con 's'. A lo largo del artículo uso la ortografía con ' $z$ ' para el nombre de la institución o citas en donde se use de esa manera y en el resto de los casos usaré la norma actual. Ver: Norma de escritura de la lengua tseltal https://site.inali.gob.mx/pdf/norma_tseltal.pdf y Norma de escritura para la lengua tsotsil https://site.inali.gob.mx/pdf/norma_tseltal.pdf (consultados el 06-8-2020).

2 Stephen E. Lewis, Rethinking Mexican Indigenismo: The INI's Coordinating Center in Highland Chiapas and the Fate of a Utopian Project (Albuquerque: University of New Mexico Press, 2018), 63-87.
} 
Primero, intentaron eliminar las connotaciones peyorativas, humillantes, racistas y discriminatorias que la palabra «indio» había adquirido. Cuando Rosario Castellanos trabajó como directora y escritora en el teatro guiñol para las campañas educativas del CCI los antropólogos promovían el uso de la palabra «indígena». Probablemente consideraron que este último término tenía una semántica neutral, al menos etimológicamente, ${ }^{3}$ y como consecuencia tendría un efecto social de mayor justicia y dignificación. Aunque, de acuerdo con Paula López Caballero, la definición de quién era indígena y cuáles sus atributos eran objeto de discusiones. El concepto se fijó socialmente de manera paulatina, sobre todo en las esferas académicas y políticas, como consecuencia de las propias acciones del INI. Mientras que la noción de «indígena» aun es discutible, ${ }^{4}$ el uso del término «indio» con sentidos peyorativos sigue vigente en diversos usos del lenguaje vernáculo.

Segundo, promovieron el uso del español entre los hablantes de tsotsil y tseltal. En diversos documentos de trabajo, señalaban que los tsotsiles y tseltales eran pueblos debilitados por la dominación y el abuso. Consideraban que a causa de eso su autovaloración, hábitos de pensamiento y conducta estaban condicionados por la sumisión. Los especialistas suponían que si los hablantes de tsotsil y tseltal aprendían a hablar, leer y escribir en español se generarían condiciones sociales y económicas para que dignificaran su vida y su percepción de sí mismos. Porque, decían que entender la misma lengua de sus opresores les daba un arma de lucha para defenderse de los engaños y solucionar los problemas que los aquejaban. ${ }^{5}$

Tercero, promovieron la literacidad de los hablantes de tsotsil y tseltal. Entre los lingüistas y sus informantes crearon textos y materiales didácticos bilingües para familiarizar a los hablantes de esas lenguas

\footnotetext{
3 El término indígena proviene de las raíces latinas inde «de allí» y gens «gente»; gente originaria de un lugar.

4 Paula López Caballero, «Las políticas indigenistas y la 'fábrica' de su sujeto de intervención en la creación del primer Centro Coordinador del Instituto Nacional Indigenista (1948-1952)», en Nación y alteridad. Mestizos, indígenas y extranjeros en el proceso de formación nacional, coords. Paula López Caballero y Daniela Gleizer (México, D.F.: Universidad Autónoma Metropolitana/Ediciones EyC, 2015), 69-108.

5 Archivo Histórico del Centro Coordinador Indigenista Tzeltal-Tzotzil (AHCCITT). Secc.: Educación, Sub.: Correspondencia, Año: 1958, Caja: 2, Exp.: 0001. Plan de trabajo para 1958. Educación. Firmado por Fidencio Montes Sánchez.
} 
con la escritura. Después de que aprendían a leer y escribir en su lengua nativa, introducían el español de forma escrita.

Los proyectos indigenistas relacionados al lenguaje se han considerado como una política más o menos homogénea y han sido acusados de promover el uso del español en las escuelas y otros espacios públicos y, con ello, causar el desplazamiento de las lenguas originarias de la población. En este trabajo propongo que se trató de iniciativas atravesadas por posiciones ideológicas, sobre las lenguas, opuestas y contradictorias entre sí. Para mostrarlo analizo textos escritos por Rosario Castellanos vinculados con su trabajo en el CCI. ${ }^{6} \mathrm{Mi}$ análisis no tiene ninguna intención biográfica acerca de la escritora, sino estudiar desde una perspectiva histórica y discursiva las voces sociales que evidencian las tensiones ideológicas en esos programas educativos.

Como se ha mostrado en otras investigaciones, ${ }^{7}$ las ideologías sobre la lengua implican percepciones sobre los hablantes, de manera que, el ordenamiento de la lengua conlleva una intención de ordenamiento social, de clase o de poder. Considero que las ideologías acerca de las lenguas tsotsil y tseltal, y sus hablantes se relacionaron de manera contenciosa en el desarrollo de los proyectos indigenistas. Esas contiendas internas trascendieron y una de sus principales secuelas es el asentamiento de la categoría «lenguas indígenas» de uso vigente en el lenguaje político y en el habla generalizada. Este trabajo advierte de los contrasentidos ideológicos que anteceden al uso de esa clasificación y propone la reflexión sobre las posibles contiendas ideológicas que puede entrañar la categoría.

\section{LOS TÍTERES EN EL MARCO DE LAS TEORÍAS, IDEALES Y PRÁCTICAS EDUCATIVAS DEL CCI}

En el Centro Coordinador Indigenista (CCI) la educación se consideró un proyecto integral donde la alfabetización estaba supeditada a la

\footnotetext{
6 Varios de estos textos fueron recopilados por el antropólogo Carlos Navarrete Cáceres, Rosario Castellanos, su presencia en la antropología mexicana (México, D.F.: Instituto de Investigaciones Antropológicas de la Universidad Nacional Autónoma de México-Programa de Investigaciones Multidisciplinarios sobre Mesoamérica y el Sureste, 2007).

7 Por ejemplo: Cooper, 1997; López García, 2015; Zabaltza 2006.
} 
utilidad práctica, ${ }^{8}$ de manera que había actividades escolarizadas y se hacían campañas educativas en las plazas públicas, en los mercados o durante los días de fiestas para difundir temas diversos y promover la alfabetización en las lenguas de la región. ${ }^{9}$

La perspectiva de los antropólogos que trabajaban en el CCI estaba influenciada por los ideales revolucionarios, el nacionalismo mexicano y la escuela de la antropología culturalista desarrollada en Estados Unidos. ${ }^{10}$ En la teoría y los estudios etnográficos de los documentos resguardados en los archivos del INI se habla sobre las cualidades de la «raza indígena» y de la interdependencia entre los indios y los no indios ${ }^{11}$ en regiones como los Altos de Chiapas donde la pequeña urbe de San Cristóbal de las Casas estaba rodeada de poblados indígenas. Para mediar entre esos dos mundos y como estrategia para introducir los programas sociales, los antropólogos del CCI reclutaron jóvenes tsotsiles y tseltales con cierto conocimiento del español que fungieron como elementos de contacto entre los pobladores y la institución. Les llamaron promotores culturales y los capacitaron como maestros, enfermeros, técnicos agrónomos, trabajadores sociales e informantes de lingüística.

En varios lugares la población se mostraba recelosa de cooperar o aceptar abiertamente las propuestas del CCI, de manera que el trabajo de los promotores era inducir la aceptación. Por ejemplo, no todos los poblados contaban con escuela; entonces, los promotores tenían la tarea de convencer a las familias de los beneficios de construir una escuela, para lo que debían donar parte de sus terrenos, material y mano de obra. En donde sí había escuela, muchos padres decidían no enviar a sus hijos, menos aún a las mujeres; en esos casos los promotores abogaban

\footnotetext{
8 Gonzalo Aguirre Beltrán, Teoría y práctica de la educación indígena (Ciudad de México: Instituto Nacional Indigenista-Fondo de Cultura Económica, 1992).

9 Stephen E. Lewis (2011, enero). «Modernizing Message, Mystical Messenger: The Teatro Petul in the Chiapas Highlans, 1954-1974». The Academy of American Franciscan History, 67(3), 375-397. Recuperado de http://muse.jhu.edu/journals/tam/summary/v067/67.3.lewis.html; Susana Ayala Reyes, "Creatividad en los márgenes del estado: Apropiación de los títeres Petul-Xun en los Altos de Chiapas» (Tesis doctoral, Departamento de Investigaciones Educativas del Cinvestav, 2019a) 32-46.

10 Navarrete, Rosario Castellanos, 19, 23; Andrés Medina Hernández «Estudio preliminar. La trama, los hilos y los nudos de un proyecto de investigación. La Universidad de Chicago en los Altos de Chiapas», en Rossana Guber, La articulación etnográfica (Buenos Aires, Argentina: Editorial Biblos, 2013), 11-34.
}

11 AHCCITT. Aguirre, Beltrán. 1955. Secc. Dirección, Sub.: Informes, Año: 1955, Caja: 2, Exp.: 0044. 
por la importancia, la necesidad e incluso la obligación legal de enviarlos a tomar clases. ${ }^{12}$ En este panorama los antropólogos que dirigían la institución hacían experimentos con actividades de difusión y entretenimiento para probar si funcionaban como medios de convencimiento o de simple entretenimiento para la población local.

Desde la década de los treinta las campañas de alfabetización, educación sanitaria y política con obras de títeres guiñol habían funcionado bien entre la población de la periferia de la Ciudad de México. ${ }^{13}$ Basados en esas experiencias los antropólogos en el CCI consideraron formar algunos de los jóvenes tsotsiles como titiriteros de guiñol en 1954. En un inicio las obras de títeres se concibieron como un recurso audiovisual para las campañas educativas. En poco tiempo las obras se convirtieron en espacios donde los promotores culturales negociaban con los pobladores su colaboración en los proyectos del INI y discutían colectivamente problemas locales; por ejemplo, pleitos entre vecinos, intrigas contra el maestro, dudas sobre cuestiones de salud, entre otras cosas.

En 1955 la precariedad en el presupuesto de la institución obligó a buscar vías económicas para realizar las actividades institucionales. Entonces las obras de títeres que ya contaban con gran aceptación por parte de la población se convirtieron en la punta de lanza con las que el CCI implementó las campañas educativas de alfabetización, salud, higiene, economía cooperativista, mejora de animales de granja y muchas cosas más. ${ }^{14}$

En ese momento fue que Rosario Castellanos ${ }^{15}$ se convirtió en escritora de los guiones para las obras de los títeres Petul y Xun (Pedro y Juan en español) que eran representadas por los promotores culturales

\footnotetext{
12 Andrés Aubry, «Les Tzotzil Par Eux-Mêmes. Récits et écrits de paysans indiens du Mexique». (París: Éditions L'Harmattan, 1988).

13 María Rosa Gudiño y Susana Sosenski, «El teatro guiñol, la televisión mexicana y la educación para la salud a mediados del siglo XX», História, Ciências, saúde-Manguinhos 24, n. ${ }^{\circ} 1$ (2017): 201-221. http://dx.doi.org/10.1590/s0104-59702016005000027 ; Elena Jackson Albarrán, «Comino Vence Al Diablo and Other Terrifying Episodes: Teatro Guiñol's Itinerant Puppet Theater». Seen and Heard in Mexico. Children and Revolutionary Cultural Nationalism (Lincoln \& Londres: University of Nebraska Press, 2014), 175-212; Juan Jiménez Izquierdo, «Entrevista con Don Ferruco», Tramoya 10, (1978): 52.

14 Lewis, 2018.

15 AHCCITT. Secc.: Educación, Sub.: Correspondencia, Año: 1958, Caja 2, Exp.: 0001. Presupuesto de educación, 1955.
} 
tsotsiles. Entre 1956 y 1957 Castellanos trabajó con el director de teatro Marco Antonio Montero, quien fue el primero en escribir los guiones para las obras. Después, Castellanos junto con el pintor Carlos Jurado se hizo cargo de la compañía tsotsil y formó la segunda compañía de titiriteros en lengua tseltal. Con los años y la fama de Rosario Castellanos como escritora algunos testigos han supuesto que ella creó a los personajes. Sin embargo, se ha mostrado que los protagonistas de las obras fueron una creación colectiva de los propios titiriteros en la interacción con el público durante las representaciones. ${ }^{16}$ La población de los Altos se apropió de este arte y sus integrantes se convirtieron en espectadores activos e interlocutores de los títeres. Años después de su auge en las campañas educativas del INI, las obras de Petul y Xun tienen presencia en la memoria de las personas. ${ }^{17}$

Según Carlos Jurado, ${ }^{18}$ él y Rosario Castellanos se convirtieron en acompañantes y a la vez en espectadores del trabajo de los titiriteros que marcaban el curso y la realización de las obras con temas basados en los guiones, pero con improvisaciones constantes. Él, en tanto artista plástico, apoyaba la fabricación de muñecos y vestuarios iguales a los que usaban las personas de cada poblado. Rosario Castellanos escribía guiones de acuerdo con las necesidades planteadas por las distintas áreas del Instituto. ${ }^{19}$

En 1956 el antropólogo Aguirre Beltrán, alto funcionario del INI y uno de los principales teóricos del indigenismo de esos años, informaba al director general del Instituto:

Se ha venido desarrollando más el recurso de agitación cultural que es el teatro de muñecos de funda regional. Su participación cada vez más activa en el programa educativo del Centro ha

\footnotetext{
16 Susana Ayala Reyes, «Creatividad en los márgenes del estado: Apropiación de los títeres Petul-Xun en los Altos de Chiapas» (Tesis doctoral, Departamento de Investigaciones Educativas del Cinvestav, 2019a).

17 Susana Ayala «Hands that Propose and Hands that Respond: Gestural Interaction in Petul-Xun Puppets Performances in Highland of Chiapas», Paedagogica Historica: International Journal of History of Education 55, no. 2 (248-250) DOI: 10.1080/00309230.2018.1521447

18 Comunicación personal. 24 de noviembre de 2016.

19 AHCCITT. Secc.: Dirección, Serie: Lingüística, Sub.: Correspondencia, Año: 1956, Caja: 1, Exp.: 0001. Informe de la dirección de lingüística.
} 
dado óptimos frutos. Actualmente se le utiliza como elemento indispensable en nuestra Escuela Abierta. Este teatro está manejado por hablantes nativos de las lenguas vernáculas de la zona y en su funcionamiento toma siempre en cuenta las características culturales de las comunidades a donde lleva su mensaje. ${ }^{20}$

Desde la perspectiva de los antropólogos y del lingüista del CCI, Carlo Antonio Castro se afirmaba que para el Teatro Petul, como se llamaba institucionalmente a la compañía de títeres, era necesario manejar el idioma nativo y no el español que en ese momento era la única lengua usada en el sistema educativo mexicano. El uso del tsotsil y del tseltal era lo que se consideraba el elemento que definía el acercamiento del teatro guiñol a la realidad que vivían los espectadores. Los directivos del CCI también señalaban que el teatro debía «aplicar detalladamente los conocimientos de todos los aspectos culturales y sociales que se tienen de las comunidades indígenas». ${ }^{21}$

En los guiones escritos por Rosario Castellanos entre 1956 y $1957^{22}$ se desarrolla un juego de opuestos y contrastes en el carácter de los protagonistas. Uno de ellos, Xun (en español Juan) desconfía o desconoce la labor de los promotores y los proyectos del INI, ignora la visión científica y occidentalizada sobre el cuidado de la salud y el cuidado del medio ambiente, el comercio, el uso del español, tiene malos hábitos de higiene, en fin, representa a una persona que, entre otras cosas, necesita estar educada. El otro personaje, Petul (en español Pedro) es todo lo opuesto, conoce el trabajo del personal del INI o él mismo es un promotor, ha sido educado en la escuela y por lo tanto tiene conocimientos variados, confía en los médicos, maestros, agrónomos, trabajadores sociales o según sea el caso. En un artículo escrito para la Revista de la Universidad de México en 1965, la escritora reflexiona sobre el carácter de los personajes:

\footnotetext{
20 AHCCITT. Secc.: Dirección, Sub.: Informes, Año: 1956, Exp.: 0020. Informes del CCITT. Educación. Por Gonzalo Aguirre Beltrán subdirector del INI, septiembre de 1956.

${ }_{21}$ Instituto Nacional Indigenista, «El teatro Petul», Acción Indigenista, Boletín Mensual del INI 24, junio de 1955.

22 INI, Teatro Petul (México: INI, 1956); INI, Teatro Petul 2 (México: INI, 1961); INI, Teatro Petul 3 (México: INI, 1962).
} 
Petul es el protagonista de las aventuras, el prototipo del hombre avisado, abierto a las noticias que le traen sus amigos mestizos o blancos; gracias a cuya intervención el desenlace resulta siempre un triunfo de la inteligencia sobre las supersticiones, del progreso sobre la tradición, de la civilización sobre la barbarie. Y la pareja de Petul es Xun, su contraparte, el indígena típico, reacio [...]. El que se niega a asistir a la escuela [...]; el que desdeña las sugerencias de los técnicos agrícolas; el que en vez de consultar a un médico [...], llama al brujo; el que no quiere prestar su ayuda para la construcción de caminos vecinales; el que no permite que se entube el agua porque es una profanación de los manantiales sagrados; el que permite que sus hijos mueran de tosferina porque los ha ocultado para evitar que fueran vacunados; el que entrega su tierra [...] porque no sabe que existe una ley que lo protege, el que encuentra en el alcohol el camino de la sabiduría, del olvido, del aturdimiento; el que lapida al que se aventura, sin defensa, hasta su paraje [...]; el que se deja esquilmar [...] y soporta el robo cotidiano el que cree que su condición es destino y su padecimiento castigo y su conformidad obediencia y su inercia virtud. Pero al final de cuentas Xun, después de todo ente de ficción, abandona sus errores redimido, no tanto por razones ni por experiencias adversas, sino por el ejemplo optimista de Petul. ${ }^{23}$

El fragmento anterior muestra dos tipos de indígenas totalmente opuestos: uno que se relaciona amistosamente con los mestizos y los blancos, inteligente, progresista, civilizado y optimista. El otro es según el texto un indígena típico, es decir, tradicionalista, bárbaro, incivilizado, reacio a la modernidad y a la ciencia. Además, supersticioso, ignorante, alcohólico y violento, al mismo tiempo resignado y sometido al abuso, entregado a la fatalidad y al padecimiento.

El artículo fue escrito siete años después de que Rosario Castellanos dejara de trabajar en el CCI. A lo largo de todo el texto reflexiona sobre su experiencia personal en la institución y describe los procesos en los que participó. La descripción que hace de los personajes parte del relato experiencial, de su percepción sobre los protagonistas y de los argumentos finales de los guiones. Si bien, las obras fueron resultado del consenso y

${ }_{23}$ Rosario Castellanos «Teatro Petul», Revista de la Universidad de México XIX, no. 5 (1965): 30-31. 
la discusión con los antropólogos y los otros participantes del CCI, estos estereotipos también pueden estar relacionados con la propia percepción de la escritora. Ella provenía de una familia terrateniente y vivió su infancia y juventud en Comitán, un pequeño centro urbano muy cercano a los Altos de Chiapas. Esto le hacía conocedora de primera mano de la discriminación y subordinación de los tsotsiles y tseltales, que había llegado a ser un sistema de semi-esclavitud con forma de peonaje. También conocía los estereotipos creados en torno a ellos, ${ }^{24}$ arraigados desde la colonia y prevalecientes mucho tiempo después.

No obstante, la escritora tenía una posición ideológicamente progresista y crítica hacia las relaciones de dominación impuestas por las desigualdades de clase social, racismo y género..$^{25}$

\section{GUIONES SOBRE LA DIFERENCIA LINGÜÍSTICA EN LA BÚSQUEDA DE LA IGUALDAD SOCIAL}

Los textos de Rosario Castellanos analizados en este trabajo tratan sobre los indígenas, sus lenguas, sus procesos de aprendizaje y comunicación. Los guiones para las obras de Petul y Xun fueron escritos de acuerdo con los planes y necesidades institucionales. En el mismo artículo escrito para la revista de la Universidad, Castellanos describe el proceso para desarrollar un guion.

Todo comenzaba al definir los temas de las obras con los jefes de las secciones del CCI:

El de Salubridad, por ejemplo, necesita iniciar una campaña contra el tifo. El de Educación está empezando a poner en práctica el sistema de la Escuela Abierta. Al de Comunicaciones le faltan brazos para abrir una brecha de penetración. Hablan conmigo, me explican los puntos esenciales del problema, me señalan

\footnotetext{
24 Juan Pedro Viqueira, «Las causas de una rebelión india: Chiapas, 1712», en Chiapas los rumbos de otra historia, eds. Juan Pedro Viqueira y Mario Humberto Ruz (México, D.F.: Universidad Nacional Autónoma de México, Centro de Investigaciones y Estudios Superiores en Antropología Social, Centro de Estudios Mexicanos y Centroamericanos, Universidad de Guadalajara, 1995), 103-143.

25 Joseph Sommers, «Forma e ideología en "Oficio de tinieblas" de Rosario Castellanos», Revista de Crítica Literaria Latinoamericana, 2, no. 7/8 (1978):73-91. DOI: 10.2307/4529870
} 
los malos entendidos [...]. Yo escribo un borrador [...] al que ellos añaden aclaraciones y suprimen ambigüedades. ${ }^{26}$

Una vez escrito el guion en español lo trabajaba junto con los promotores tsotsiles y tseltales para tener versiones en cada lengua:

El primer paso es traducir [...]. Acabamos de entendernos a fuerza de ademanes, de perífrasis, de entonaciones de la voz. En última instancia recurrimos al lingüista para que apruebe la exactitud de la traducción. ${ }^{27}$

Ese proceso tenía la dificultad de que los promotores apenas hablaban español, mientras que Castellanos solo conocía algunas palabras sueltas de las lenguas tsotsil y tseltal, de manera que las traducciones se hacían con explicaciones apoyadas en recursos paralingüísticos. Los guiones pasaban por varias revisiones hasta que todos quedaban conformes con el contenido. El lingüista del Centro Coordinador, Carlo Antonio Castro, apoyaba ese proceso para lograr las versiones finales en las lenguas que no eran comprensibles para Castellanos, ni para los jefes de sección; con todo, es importante resaltar que la comunicación se lograba mediante recursos comunicativos diversos aun cuando los participantes hablaran lenguas distintas. Por otra parte, los titiriteros no memorizaban los guiones así que, en la realización, las obras eran improvisadas en la lengua de cada comunidad. Contrario a lo que podría suponerse la improvisación constituye un recurso lingüístico y cultural que en ese caso enriqueció las funciones poéticas y lúdicas de las obras, sin que se perdiera el mensaje establecido en los guiones. ${ }^{28}$

Los guiones para las obras de títeres que escribió Rosario Castellanos entre 1956 y 1957 estuvieron orientados a dar consejos sobre higiene, salud, mejora de animales de granja y alfabetización, en ellos se combaten el alcoholismo y las creencias en enfermedades causadas por brujos y otros seres sobrenaturales. Las historias insisten en los beneficios de asistir a la escuela de manera regular y en la buena voluntad

\footnotetext{
26 Rosario Castellanos «Teatro Petul», Revista de la Universidad de México XIX, no. 5 (1965): 30-31.

27 Castellanos en Navarrete, «Teatro Petul», 2007, 123.

28 Susana Ayala Reyes, «Creatividad en los márgenes del estado: Apropiación de los títeres Petul-Xun en los Altos de Chiapas» (Tesis doctoral, Departamento de Investigaciones Educativas del Cinvestav, 2019a), 85-91.
} 
política del INI al crear cooperativas comerciales, puestos médicos, escuelas y campos agrícolas. Se trata de textos para la formación de ciudadanos, algunos guiones tienen un carácter abiertamente nacionalista como se muestra en el siguiente fragmento de «La Bandera»:

Petul: Sí, todos vivimos a la sombra de nuestra bandera, lo mismo que los hijos viven alrededor de su madre.

Xun: Entonces, Petul, a nuestra bandera debemos quererla y respetarla lo mismo que a nuestra madre [...] todos los días.

Petul: Todos los días; y trabajando mucho, para que nuestra madre, para que nuestra patria, para que México, sea más rico y ya no haya ni uno solo de nosotros que tenga hambre y no pueda comer, que tenga frío y no pueda vestirse. Todos debemos vivir mejor y por eso tenemos que trabajar más.

Xun: Pero también tenemos que aprender más.

Petul: Sí, para que nuestra madre, para que nuestra patria, para que México, no tenga hijos ignorantes, hijos de los que se puedan burlar los que saben más de ellos.

Xun: Sí, Petul, todos queremos ser más felices y más buenos, para que no sufra nuestra patria, nuestra madre, México. ${ }^{29}$

Otros dos, presentan como tema principal a los expresidentes mexicanos Benito Juárez y Lázaro Cárdenas como los que sentaron las bases de los derechos civiles de los indios. En todos los guiones se desarrolla una trama que muestra posibilidades para mejorar las condiciones de vida de la población y se abordan de distintas formas los derechos vinculados a la igualdad social de la población. Es particularmente notorio el énfasis sobre el conocimiento que se gana al aprender a hablar español. Esto se aprecia en el fragmento del guion que narra la historia de Benito Juárez, la historia recalca la idea de que el protagonista logró ascender socialmente y ser presidente de México en primer lugar porque aprendió a hablar «castilla», como se le llamaba en las zonas rurales al idioma español.

29 Carlos Navarrete. Rosario Castellanos, 54. 
Benito: Soy indio y no tengo por qué avergonzarme de mi raza. Pero ser indio no quiere decir ser tan infeliz como nosotros.

Bernardino: ¿Y qué querías? ¿Ser igual que las gentes de razón?

Benito: Sí, saber lo que saben los ladinos. ${ }^{30}[\ldots]$ Yo quiero aprender a hablar castilla y a leer.

En otra escena cuando Benito Juárez logra asistir a la escuela:

Benito: He aprendido a hablar bien el castellano; ya sé leer y escribir y hacer cuentas. Ahora ya ninguna gente de razón puede engañarme porque sea yo un ignorante, ya nadie me puede despreciar.

María Josefa: ¡Si vieras hermano, qué envidia te tengo!

Benito: Pero mi gusto no es cabal, porque pienso en ti, en la gente de mi pueblo que no habla más que zapoteco y que no sabe nada. Pienso en todos los indígenas del estado de Oaxaca y de México entero. Tan pobres, tan ignorantes.

La historia de Benito Juárez se desarrolla sobre la misma oposición entre el indio que aprende español y con ello adquiere conocimientos y un lugar socialmente digno, y el indio pobre e ignorante porque solamente conoce la lengua de su pueblo. Los guiones para las obras de Petul y Xun despliegan la misma oposición y la premisa de que hay un problema de carácter social que puede resolverse si se transforma el uso del lenguaje.

En la introducción a la primera compilación de los guiones del Teatro Petul ${ }^{31}$ publicada por el INI, Rosario Castellanos explica que la

\footnotetext{
30 «Ladinos» es una categoría social usada en los Altos de Chiapas. Su significado es polisémico. En este fragmento y en la mayoría de los guiones escritos por Rosario Castellanos, el término se usa para referirse a los mexicanos que no son considerados indígenas. Otro significado del término vigente desde antes de la década de 1950 es para referirse a los indígenas que han renegado de su raza, dejan de hablar las lenguas de sus comunidades de origen y se visten con ropa del estilo urbano, tratando de asemejarse a los no indígenas. En los archivos del INI son frecuentes las historias de ladinos, que abusaban de los indígenas, aunque fueran del mismo pueblo. Actualmente el término se sigue usando, aunque su semántica es diversa y confusa porque siempre depende de la situación en la que se usa, quién la usa y a quién designa. Ver. Pitarch, 1995.
}

31 INI, Teatro Petul, 1956. 
intención de representar a los personajes en esas oposiciones es didáctica, ya que cada guion aborda directamente un problema y prescribe una solución. ${ }^{32}$ En estos guiones cada uno de los personajes representa un conjunto de características ideales y cerradas. Xun está en el mundo de los problemas y Petul en el de las soluciones, la frontera entre ellos es muy clara y Castellanos presenta a cada personaje como un sujeto discursivo, correspondiente a una unidad de sentido definida, con un hacer y un destino marcado de antemano. En los guiones Xun es una víctima rescatada por el héroe Petul. Este tipo de personajes tan concluidos en sí mismos tienen ciertas correspondencias con algunas características señaladas por Bajtín respecto al género épico: ${ }^{33}$

[...] la tradición nacional (no la experiencia personal y el pensamiento libre que la genera) sirve como fuente de lo épico $[\ldots]$

El pasado épico se llama «pasado absoluto» por una buena razón: es a la vez monocrónico y valorizado (jerárquico); carece de relatividad, es decir, de progresiones graduales puramente temporales que puedan conectarlo con el presente.

Los personajes están basados en ideas tradicionales sobre los sujetos sociales a los que se refieren, las obras se desarrollan en un tiempo muy definido, no precisamente el pasado, pero sí un tiempo que carece de relatividad. El pasado de los indígenas se condensa en Xun y su futuro en Petul. Lo anterior se confirma con la explicación de Rosario Castellanos sobre la intención de que los textos buscaban convencer a los indígenas, según sus propias palabras, para «hacerles recuperar la memoria de su dignidad y erguirlos e inquietarlos y hacerlos moverse [...] en un terreno desconocido: el de la igualdad». ${ }^{34}$

Considero necesario insistir en que los guiones no se representaron tal como fueron escritos sino echando mano de complejos recursos de improvisación. Aunque el análisis de la representación improvisada

\footnotetext{
32 Castellanos en INI,1956, 4.

33 M. M. Bakhtin «Epic and Novel», en The Dialogic Imagination Four Essays by M. M. Bahktin (Austin: University of Texas Press, 1981), 13, 15.

34 Castellanos en Navarrete, 2007, 121.
} 
excede este trabajo, es importante señalar que las dimensiones épicas de los personajes son características de los guiones escritos por Rosario Castellanos y no de la ejecución de las obras. El siguiente fragmento del guion Petul y Xun juegan a la lotería ${ }^{35}$ está enfocado en la utilidad social de aprender español y cómo eso abriría una de las puertas de la igualdad.

\section{Petul y Xun juegan a la lotería}

Petul: Ven Xun. Vamos a jugar a la lotería.

Xun: ¿A la lotería? Yo no sé qué juego es ese.

Petul: Yo sí. Y a veces he ganado. ¿Te acuerdas de esa alcancía donde guardo mis ahorros? Pues la gané en la lotería.

Xun: Enséñame cómo se juega, Petul. Yo también quiero tener una alcancía como la tuya.

Petul: Para este juego se necesitan por lo menos dos gentes. Una pongamos que eres tú, tiene un cartón con varias figuras dibujadas. Figuras de animales, de árboles, de flores, de muebles. Y yo tengo en la mano recortadas como barajas y las voy sacando una por una y digo: «El arpa suena bonito», «el conejo correlón». El Instituto hizo muchos juegos de lotería y los repartió entre los promotores de Educación.

Xun: Vamos a jugar Petul. Tú eres el maestro y vas diciendo los nombres de las figuras y yo las voy señalando en mi cartón.

Petul: «La cama para dormir», «El maíz que sembró mi padre», «La vaca lechera».

Xun: Dime los nombres en tzeltal, Petul. No entiendo bien el castellano.

Petul: Te voy a decir los nombres en tzeltal; pero fíjate que detrás de cada tablita con nombre en bats'ilc'op ${ }^{36}$ está cómo se

35 He eliminado algunas partes del guion para adaptarlo a este artículo.

36 Nombre de la misma lengua que también en español es llamada tseltal. 
llama en castellano. Así, a fuerza de mirarlo y de repetirlo en el juego, lo vas a aprender.

Xun: Y si gano ¿cuál es el premio?

Petul: El premio, Xun, es que vayas entendiendo y hablando el castellano. Que sepas lo que te dicen los ladinos y que contestes bien las palabras de los caxlanes. ${ }^{37}$ Que cuando platiques con ellos, cuando hagas tratos, te respeten como su igual.

En este guion donde se explica cómo usar la lotería para aprender español, también se muestra la parte ideológica sobre las ganancias comunicativas y sociales que implica entenderlo y hablarlo. Saber lo que decían los ladinos, era imprescindible para evitar el abuso y el engaño; en cambio, contestar bien las palabras de los caxlanes implica relacionarse con cortesía, con educación, con claridad y con la comprensión suficiente para poder hacer tratos comerciales y de servicios sin terminar en desventaja. El texto es consecuente con las ideas acerca de que la discriminación está en el desconocimiento total y parcial del lenguaje. La frase «que contestes bien» significa que se privilegia el conocimiento de las formas gramaticalmente correctas de la lengua por encima de las posibilidades comunicativas con formas gramaticalmente incorrectas. Es decir, aunque los tsotsiles y tseltales pudieran usar diversos recursos de comunicación para interactuar con hablantes de español, no se consideraba suficiente si no usaban los formalismos relativos a aspectos como la pronunciación o el uso de ciertas palabras. Tal como se ha señalado en el estudio de María López García, ${ }^{38}$ mediante la idea de obtener un premio, se inducen e internalizan prejuicios sociales sobre las lenguas, además de emociones encontradas con relación a las lenguas no prestigiadas. La idea que subyace es que si se cambia la lengua, si se usa de manera adecuada la misma lengua de los ladinos y los caxlanes, entonces habría una recompensa.

\footnotetext{
37 Término común con que los tsotsiles y tseltales nombraban a la gente no indígena, de clase alta. Es una categoría social que también se ha usado como sinónimo de «blancos» en el sentido de discriminación racial.

38 María López García, "Aprendan el "vosotros” para hablar con los españoles en su idioma: La identificación lingüística en la Argentina como tensión entre el orgullo y la minusvaloración», Historia y Memoria de la Educación, 2 (2015): 105. DOI:10.5944/hme.2.2015.14234
} 
Los guiones plantean un conjunto de problemas sociales que se proponen resolver con soluciones lingüísticas. En el caso del guion sobre la lotería, se plantea un conflicto de clase que data de siglos atrás y que era bien conocido por la escritora en la cotidianidad de una parte de su vida. Desde la teoría de Bajtín sobre los géneros discursivos, los guiones pueden considerarse enunciados, entre otras cosas porque constituyen unidades de la comunicación discursiva. En palabras del autor

... un enunciado completo y concluso (réplica del diálogo) [...] se enfrenta de manera directa e inmediata a la realidad (al contexto extraverbal del discurso) [...]. Una obra es eslabón en la cadena de comunicación discursiva [...] se relaciona con otras obras-enunciados $[\ldots]$ a los que contesta y con aquellos que le contestan a ella [...]. [El] segundo rasgo es la conclusividad específica del enunciado [...]. El primero y más importante criterio de la conclusividad es la posibilidad de ser contestado. $\mathrm{O}$, en términos más exactos y amplios, la posibilidad de tomar una postura de respuesta en relación con el enunciado. ${ }^{39}$

Esos guiones, tal como dice Bajtín con respecto del enunciado, están insertos en una larga cadena de enunciados, es decir, forman parte de un devenir histórico de interacciones y de luchas en donde se entraman pensamientos y posturas del propio enunciador, en este caso de Rosario Castellanos, aunque también se entretejen los pensamientos y posturas de otros, como es el caso de los jefes de sección del CCI, los antropólogos y el lingüista mencionado en este texto.

Esta enunciación es un posicionamiento ante las situaciones extraverbales, entre las que se cuentan los conflictos sociales. El guion está enlazado históricamente en las interacciones y luchas implicadas con los enunciados que previamente se hayan emitido desde las instituciones del gobierno, los ladinos, los indígenas o la misma vida personal de Rosario Castellanos cuando tuvo contacto con todos esos elementos. Aunque la autoría de estos guiones es de ella, los textos se encuentran

39 Mijaíl Mijáilovich Bajtín, Estética de la creación verbal (México, D.F.: Siglo XXI, 1979/2009), 262, $263,265$. 
atravesados por las subjetividades de todos los que intervinieron en su creación.

En el guion sobre la lotería se muestra que el CCI proponía abiertamente el bilingüismo en las actividades educativas, eso constituyó una lucha ideológica y política entre los funcionarios del INI con los representantes de la escuela rural impulsada por la Secretaría de Educación Pública, en donde se negaba el uso de las lenguas originarias en la instrucción escolarizada. En la obra, el títere Petul como maestro sí permite que Xun use su lengua originaria al motivarlo a leer tarjetas de ilustraciones que tienen escritos los nombres de las cosas en forma bilingüe. Por otra parte, despliega el argumento de la ganancia ligando dos ideas contradictorias con relación al tseltal; hablarlo está bien porque es la lengua en la que Xun comprende mejor, pero las ventajas sociales y económicas son resultado de hablar el español.

Además, hay otra trama de relaciones sociales evidente en el guion. Al mencionar a los ladinos y los caxlanes se usan dos categorías sociales en las que se mezclan ideas de clase y raza, que se propone pueden hacerse menos conflictivas y más igualitarias si son los indígenas quienes aprenden la lengua de los otros.

La igualdad entre personas de distintos orígenes fue tema de otro guion titulado Petul y el diablo extranjero, en este texto se tratan las actitudes e ideologías racistas de los propios tsotsiles y tseltales hacia los blancos y los negros. Este guion constituyó una respuesta a hechos violentos que tuvieron lugar en 1956 en contra de turistas extranjeros que, sin conocer las normas de las comunidades de los Altos de Chiapas, se introdujeron en áreas naturales consideradas sagradas y fueron agredidos, como resultado un extranjero fue asesinado. ${ }^{40}$ Sumado al racismo por el color de piel, se aborda también la discriminación hacia las lenguas desconocidas. Así la reflexión sobre las lenguas que propuso Rosario Castellanos en sus guiones no estaba ceñida a la relación desigual entre el español y las lenguas de México.

40 AHCCITT. Secc.: Dirección, Sub.: Informes, Año: 1956, Caja: 2, Exp.: 0017. Informe de actividades del mes de septiembre de 1956. Dirigido a Sr. Prof. Alfonso Villa Rojas. Director del CCITT. Firmado por Carlos Jurado encargado de la secc. De Ayudas Audiovisuales. Fecha 7 de octubre de 1956, Las Casas, Chis. 
Petul y el diablo extranjero

(Xun va caminando muy contento. Lleva un azadón en la mano, cuando sale otro personaje y se topa con él. El atarantamiento del golpe impide a Xun advertir que el recién llegado es pelirrojo, viste estrafalariamente y habla una lengua incomprensible.)

Xun: Ay, hermano por poco me haces caer al suelo.

El extranjero: asdfgñkj qwertyuiop

Xun: (Fijándose por primera vez en el otro). ¿Qué?

El extranjero: zxcvb mn

Xun: (Santiguándose) ¡San Jerónimo bendito! Si bien me decía mi mujer que yo no saliera porque se me iba a aparecer el ijcal (sic). ${ }^{41} \mathrm{Ay}$, no, padrecito, no me hagas daño. Te juro que me voy a portar bien.

El extranjero: qrstwe, poiuy

Xun: (Empezando a enojarse) Ya basta, no estés allí echándome maldiciones.

El extranjero: ASDFGHJLN'\#\$\%\&=()

Xun: Aguarda. Tú no eres un ijcal. Mi tata me dijo que el ijcal es negro, bien negro y tamañito así. Tú eres blanco, más blanco que los ladinos de Jobel. ${ }^{42} \mathrm{Y}$ hablas una castilla ${ }^{43}$ más enrevesada que la de ellos.

El extranjero: (Se acerca a Xun y trata de tocar su vestido) zxcvb

\footnotetext{
41 El término correcto en las lenguas mayas tsotsil y tseltal es jik'al. Es el nombre que se da a un personaje de las historias de ficción originadas en los Altos de Chiapas. El jik'al es negro y tiene atributos que pueden ser de demonios u otros seres sobrenaturales. Las historias sobre el personaje pueden tener su origen en los esclavos negros escapados de las fincas cafetaleras que al ser fugitivos solo salían de noche y asaltaban a los habitantes. Ver Antonio Gómez Gómez, El Negro cimarrón. Ya'Yejal J-Ik'al (San Cristóbal de las Casas: UNAM, 2000), 1-2.

42 Nombre que dan los tsotsiles y tseltales de los Altos de Chiapas a la ciudad de San Cristóbal de las Casas.

43 En muchos documentos de las décadas de 1940 a 1960 se escriben las referencias al español como castilla o castellano.
} 
Xun: No, no te acerques

El extranjero: (Insiste en tocar al indígena) asdfgñlkjh qwertpoiuy

Xun: Que me sueltes te digo. Eres un pukuj ${ }^{44}$ bueno o malo? Eres un ángel que viene a traernos beneficios o un demonio que viene a hacernos daño?

El extranjero: Asdf Ñlkjh

Xun: No, así no nos entendemos. Mira, vamos a hacer un trato. Si eres ángel bueno, acércate. Pero si eres demonio ¡fuera de aquí!

(Xun hace un gesto tan amenazador, que el extranjero se asusta y se retira un poco. Esto lo interpreta Xun como una respuesta).

Ah, con que eres un demonio. Pues ahora mismo te voy a dar tu merecido (Xun descarga el azadón sobre el extranjero quien grita y trata de defenderse. En ese momento aparece Petul)

Petul: (Intentado separarlos) Ya, estense quietos, díganme qué sucede aquí.

El extranjero: asdfgñlkjm nmchgd jhuytiop

Xun: Mentiras, son mentiras lo que está diciendo. No te acerques Petul. Es un pukuj, es demonio y te va a hacer daño. ¿Qué no lo ves? su pelo es rojo como el fuego.

Petul: Yo he visto gente con pelo rojo. En Jobel, en Tuxtla, en México. Antes yo también me asusté y pregunté con los maestros, con la gente de razón. Me dijeron que son personas como nosotros pero como su tierra está muy lejos de aquí les dicen extranjeros.

Xun: Extranjeros.

Petul: Sí, Xun. Quiere decir que no son mexicanos. Me contaron también que hay extranjeros que son negros. Pero no por eso vamos a creer que es un ijcal.

${ }_{44}$ Personaje mítico. Demonio o diablo. 
Xun: ¿Y dónde viven? ¿ ¿Y qué vienen a buscar hasta aquí?

Petul: Vienen a pasear. Oyeron decir que México es muy bonito y quisieron venir a conocerlo.

Xun: Y por qué no lo dicen cuando se les pregunta? Yo le estuve preguntando a este quién era y que hacía. ¿Pero acaso me contestó?

Petul: No te contestó porque no entendió lo que dijiste; no sabe hablar nuestro idioma.

Xun: ¿No entiende nuestro idioma? Ja, ja. Aquí lo entienden hasta los niños chiquitos. ¿No crees, Petul, que la gente de otra parte, los extranjeros son muy tontos?

El extranjero: Asdfg ñlkjh zxcvb qwert

Xun: ¿Qué dice Petul?

Petul: No entiendo su idioma, Xun. Tú tampoco. Parece que estamos resultando tan tontos como los extranjeros.

Xun: Ese hombre me quería agarrar mi cotón,45 Yo por eso le empecé a pegar.

Petul: Muy mal hecho, Xun. Estaría mirando cómo es la tela. Tal vez lo quería comprar. A los extranjeros les gusta llevar recuerdos de los lugares por donde pasean. Si un extranjero te quiere robar algo o cometer un abuso, no debes pegarle. Hay que llevarlo con las autoridades. Y si el extranjero es malo...

Xun: Ah, entonces también hay extranjeros malos.

Petul: Sí, Xun. Hasta en eso se parecen a nosotros, en que hay unos buenos y otros malos. Aunque sean de distinto color y hablen distinto idioma son gentes como nosotros. No son ángeles ni diablos ni ijcales. Son gentes, igual que tú y que yo.

${ }_{45}$ Camisa larga de lana o algodón tejido en telar. Es una de las prendas de vestir tradicionales de los hombres en los Altos de Chiapas. 
Xun: Ahora sí ya entendí, Petul. Gracias (Haciendo una reverencia de cortesía al extranjero). Adiós señor. Que le vaya bien, que tenga usted buen viaje.

El extranjero: (Correspondiendo la reverencia con otra) asdfg ñlkjh poiuy qwert.

En este guion se aborda la alteridad a partir de la incomprensión acerca de quién es el otro y los prejuicios que surgen a partir del desconocimiento y la ignorancia de la diversidad entre los seres humanos. También hay un sesgo que señala de forma negativa las creencias hacia los seres sobrenaturales y las prácticas rituales relacionadas con esas creencias. Con relación a la diversidad lingüística hay una valoración positiva de las lenguas aun cuando sean incomprensibles y un énfasis en que el hecho de no comprender una lengua no significa ser tonto. En ninguno de los textos se dice explícitamente que alguien sea tonto por hablar una lengua originaria, sin embargo, he mostrado que con frecuencia se asocia la ignorancia con el desconocimiento del español. El guion apunta a un posicionamiento ideológico acerca de las lenguas como igualmente válidas e importantes, como parte natural de las diferencias culturales entre los grupos humanos y por otra parte subraya que hay valores morales y formas de actuar comunes a todas las personas. La afirmación de Petul «son gentes igual que tú y que yo» corresponde en su posicionamiento con la enunciación ideológica de Rosario Castellanos en una entrevista donde se deslinda de la corriente de literatura indigenista. Señala que el mayor defecto de esa corriente es su simplismo porque considera al mundo indígena como exótico y sus habitantes como víctimas. Castellanos explica que desde su punto de vista:

Los indios son seres humanos absolutamente iguales a los blancos, solo que colocados en una circunstancia especial y desfavorable. Como son más débiles, pueden ser más malos, violentos, traidores e hipócritas que los blancos. Los indios no me parecen misteriosos ni poéticos, lo que ocurre es que viven en una miseria atroz. ${ }^{46}$

\footnotetext{
46 Castellanos en Navarrete, Rosario Castellanos, 41.
} 
En este fragmento el plano de la igualdad de los indios se basa en el hecho de ser humanos, tal como en el guion anterior. Esto corresponde con la imagen de la escritora de ideas progresistas y posturas que defendieron la justicia y la igualdad social. Sin embargo, señala que el hecho de estar en circunstancias desfavorables a causa de otros, potencializa características negativas en ellos al punto de que afirma una desigualdad, no solo en cuanto a las circunstancias sociales y económicas, sino en cuanto al carácter mismo de los indios como seres humanos.

\section{DISCURSOS SOBRE LA DIFERENCIA: CONTRASENTIDOS EN LA EDUCACIÓN INDIGENISTA}

El artículo escrito para la revista de la Universidad en 1965 dirigido a un público intelectual, pone en tensión la postura ideológica que, a primera vista, parece tan asentada en los guiones. Primero, Rosario Castellanos menciona brevemente por qué usaba el término indígena en lugar de indio: "Con ellos, con los indios (aunque para evitar esta palabra que había llegado en nuestro medio a equivaler a un insulto les llamábamos oficialmente los indígenas) nuestro problema era el mismo que el del angustiado hombre contemporáneo: el de la incomunicación». ${ }^{47}$ Se trata de una reivindicación social de las personas y se pretende resolver con el uso de otras palabras. Este cambio en el lenguaje fue motivado por los antropólogos al considerar que el uso de términos diferentes conllevaría la transformación de las ideas y comportamientos hacia la población categorizada. No obstante, en otros textos no oficiales Rosario Castellanos continuó usando el término indios, lo que indica que el cambio de nominaciones fue gradual y en algunos casos no trascendió más allá del uso políticamente correcto. Hasta cierto punto esto explica la pervivencia del término indio en esferas no políticas ni académicas.

Esto da lugar a cuestionar si acaso los antropólogos, los educadores o la escritora que con frecuencia usaron la palabra indios en discursos no oficiales consideraban que podían permitírselo porque ellos se veían a sí mismos como no discriminadores, mientras impulsaban la popularización de la palabra indígena en todos los otros a quienes sí percibían

47 Castellanos, «Teatro Petul», 30. 
como discriminadores. También surge el cuestionamiento acerca de la posibilidad de que la semántica del menosprecio se deslizara más de una vez en el uso del nuevo término o en otras construcciones enunciativas. En este caso, como en otros esfuerzos documentados por planificar el uso del lenguaje con fines de transformación social, ${ }^{48}$ parece que la idea de usar una lengua neutra responde al mito de la igualdad social y de la fluidez en la comunicación entre grupos sociales distintos. Sin embargo, al expresar que el problema de incomunicación es el mismo que con aquellos que la escritora define como «el hombre contemporáneo» parece perder sentido la urgencia de aprender hablar español. ¿Si no se resolvía el problema de la comunicación, qué se buscaba con modificar el comportamiento lingüístico?

Se suponía que en el uso de indígena como nuevo término se dignificaría a las personas y la palabra se llenaría de una semántica que connotara las cualidades positivas de toda esa población. Con el paso de los años y la popularización del término indígena el problema semántico y las consecuencias sociales que acarrea corresponden totalmente con las ideas discriminatorias y racistas desarrolladas en México acerca de aquellos a quienes se llamaba indios desde la época colonial. Las historiadoras Paula López Caballero y Ariadna Acevedo-Rodrigo ${ }^{49}$ (2018) observan con respecto a la categoría de indígena que:

Cuando consideramos a las personas a quienes usualmente agrupamos o quienes se agrupan a sí mismos bajo la categoría de indígenas, necesitamos preguntar ¿qué están haciendo más allá de la identificación que esta categoría induce? También ¿cuál es la historicidad de la categoría en sí, más allá de las personas a quienes así designa?50

Con base en el planteamiento de López Caballero y Acevedo-Rodrigo es evidente que el uso del nuevo término no reivindica de ninguna forma

\footnotetext{
48 Robert L. Cooper, La planificación lingüística y el cambio social (Madrid: Cambridge University Press, 1997), 24-30; Xabier Zabaltza Pérez-Nievas «Lengua y clase» en Historia de las lenguas y los nacionalismos (Barcelona: Gedisa, 2006), 125-137. María López García, "Aprendan el "vosotros"».

49 Paula López Caballero y Ariadna Acevedo-Rodrigo, «Introduction: Why Beyond Alterity?», en Beyond Alterity. Destabilizing the Indigenous Other in Mexico, eds. Paula López Caballero y Ariadna Acevedo-Rodrigo (Tucson: The University of Arizona Press, 2018), 3-28.

50 López Caballero y Acevedo-Rodrigo, «Introduction», 19. Traducción propia.
} 
las características atribuidas a Xun ni su carácter de indígena típico. Tampoco cambió aspectos fundamentales de la percepción de Rosario Castellanos sobre los titiriteros con los que trabajaba. En el siguiente fragmento, parte del texto "Teatro Petul», la escritora describe su trabajo y expresa sus ideas acerca de ser maestra de los titiriteros y construir los guiones junto con ellos. El posicionamiento ideológico de este fragmento abre tensiones con los otros textos escritos por Castellanos:

Era preciso decirles todo: que eran personas humanas, que su patria era México, que el viento no estaba encerrado en una cueva, que los microbios existían y eran dañinos, que el hambre no constituía un estado natural sino era un mero accidente. Era preciso decirles todo, ¿pero en qué idioma? Los de ellos, dialectos derivados y envilecidos del maya, eran conocidos nada más por los especialistas en lingüística. Y estos eran escasos -para no decir que solo había uno- [...] [Un lingüista] debía bastar para adentrarse en las infinitas variantes dialectales con que rompían la unidad primitiva de su lengua los ciento diez mil habitantes de la zona sobre los que ejerce vigilancia el Instituto. Uno debía bastar para reducir tales variantes a reglas gramaticales fijas, para elaborar cartillas de alfabetización, para sorprender los mecanismos de pensamiento y representación de quienes eran -si, éste es el término exacto- nuestros antagonistas. ${ }^{51}$

Aunque en el fragmento se expresa el punto de vista personal de Rosario Castellanos, los estudios de Voloshinov y Bajtín ${ }^{52}$ proponen que el punto de vista personal es una construcción dialógica, esto quiere decir que implica la presencia de dos o más voces sociales que interactúan entre sí y pueden tener posiciones contradictorias. En los actos discursivos se enlaza la valoración del hablante sobre sí mismo, sobre el oyente y sobre aquellos de quienes habla. Esas valoraciones pueden no ser conscientes, pero están formadas de los intercambios y condicionamientos sociales e ideológicos de quien habla. En el artículo dirigido a los lectores universitarios, justo después de explicar cómo se las arreglaban ella y los titiriteros para traducir los guiones, Rosario Castellanos escribe:

\footnotetext{
51 Castellanos en Navarrete, Rosario Castellanos, 120, 21.

52 Valentín Nikoláievich Volóshinov y Mijaíl Bajtín, La construcción de la enunciación (Buenos Aires: Editorial Almagesto, 1930/1978).
} 
No puede exigírsele a esta gente tan poco acostumbrada al ejercicio de sus potencias intelectuales, que memorice sus parlamentos. Además, sería inútil. A la hora de la representación hay que improvisar para responder a emergencias no previstas. Basta con que entiendan la idea principal y con que sigan la línea general de su desarrollo. ${ }^{53}$

¿Qué significa estas aseveraciones en cuanto a la valoración que tenía Castellanos de los tsotsiles y tseltales con los que trabajaba? Contradictoriamente, hacia el final del guion donde Petul y Xun juegan a la lotería, la propuesta es fijarse en los nombres en castellano, para aprender español a fuerza de mirarlos repetidamente, esto significaría por memorización. Aunque los guiones fueran ficción, sugerían soluciones que supuestamente consideraban posibles en la práctica real de la vida de los tsotsiles y tseltales. Esto parecería ser una contradicción en cuanto a las ideas sobre las posibilidades intelectuales que tenían los locales para la memorización de una nueva lengua. ¿Esto sería suficiente para decir que Rosario Castellanos estaba condicionada social e ideológicamente por su estatus de clase? Considero que no.

Hay otro texto en el que Castellanos se refiere a los promotores que se dedicaban a ser titiriteros y explica que todos entendían y hablaban español, aunque sea de manera básica. Señala que se notaba su bajo nivel de instrucción, pero describe cómo los titiriteros aprendían cosas nuevas durante el poco tiempo libre que tenían, hace un recuento de las cosas que leían y las actividades educativas de los propios titiriteros para adquirir conocimientos de todo tipo. Añade que se despertó en ellos el interés y la ambición de aprender, tanto que ellos mismos solicitaron tener un curso de mecanografía y buscaban aprender cosas nuevas por su cuenta. ${ }^{54}$ En este caso la enunciación sobre los titiriteros contradice la idea antes expuesta sobre las pocas facultades intelectuales de los mismos que no lograban aprender los diálogos de memoria.

Ante esta comparación de enunciados contradictorios pero realizados por la misma persona, ¿cómo se puede caracterizar la postura de Rosario Castellanos en la construcción de la política indigenista del INI

\footnotetext{
53 Navarrete, Rosario Castellanos, 123, 24.

54 Castellanos en Navarrete, Rosario Castellanos, 117, 18.
} 
en los dos años que trabajó allí? ¿Los discursos personales de la escritora reflejan los posicionamientos de la institución? De acuerdo con Dorothy Holland y Jean Lave, ${ }^{55}$ la historia de las personas y de las instituciones no son equivalentes de manera inmediata. No es posible equiparar las posturas personales de la escritora con el desarrollo de las posturas de la institución. No obstante, las dos historias coinciden en la práctica local que enfrentaron en las campañas educativas mediante las obras de títeres. En esa construcción se dieron prácticas contenciosas no necesariamente solo entre personas o entidades definidas, sino entre las ideas y los posicionamientos de los actores. El interés de mostrar los textos de Rosario Castellanos y evidenciar las tensiones, contradicciones y oposiciones es enfocar los deslizamientos semánticos en el proyecto de educación del CCI.

El objetivo no es señalar a Rosario Castellanos como incoherente o racista, considero que sus aseveraciones acerca de la igualdad son tan genuinas como aquellas en donde usa términos que podrían interpretarse como discriminatorios. Las tensiones expuestas buscan señalar que las oposiciones y contradicciones están presentes en todas las trayectorias institucionales y personales. Que no hay discursos puros donde se expongan enunciados con sentidos unitarios. Como se ha hecho evidente en la exposición una misma persona puede producir enunciados distintos, con sentidos distintos, con matices que exponen contiendas ideológicas. Esto que parece obvio, apunta hacia la complejidad de cómo se construyen los posicionamientos políticos e ideológicos y cómo en el lenguaje se producen y se negocian las identidades y las relaciones sociales entre los actores presentes en el discurso. También se hace evidente que la relación entre la lengua, la ideología y la realidad social no es unidireccional.

La afirmación sobre las lenguas habladas por los habitantes de los Altos de Chiapas acerca de que son dialectos derivados y envilecidos del maya, parecen opuestas a las ideas expresadas sobre el valor de cada lengua. La palabra dialecto, está usada en un sentido que resta valor a las lenguas, aunque adelante dice que la existencia de los dialectos

\footnotetext{
55 Dorothy Holland y Jean Lave, «History in Person: An Introduction», en History in Person. Enduring Struggles, Contentious Practices, Intimate Identities, eds. Dorothy Holland y Jean Lave (Santa Fe: School of American Research Advanced Seminar, 2001), 3-33.
} 
rompe la unidad primitiva de las lenguas, dando la idea de que la existencia de los dialectos las hiciera menos primitivas. Otra pista sobre las ideas con respecto de las lenguas es, que solo podían ser conocidas por especialistas en lingüística, es decir que su dificultad radicaba en su desorden natural y la falta de orden gramatical que solo el lingüista podía encontrar y simplificar. Este fragmento, en donde se expresan ideas negativas sobre las lenguas de los titiriteros me conduce a pensar en cuánto se han asentado en el uso del término lengua indígena. Esa forma de nombrar a todas las lenguas originarias de México, parece haber cobrado fuerza en el transcurso de los años setenta y tratarse de una consecuencia de la popularización de la palabra indígena.

Actualmente se usa con frecuencia y naturalidad el término «lengua indígena» para referir una, algunas o la gran diversidad de lenguas que se han hablado en México por distintos grupos sociales desde la época prehispánica. En el ámbito educativo el uso de este término es vigente, aunque probablemente por lo difuso o polémico que puede ser el uso de indígena en los últimos años también se han usado las terminologías lenguas mexicanas o lenguas originarias. De acuerdo con Paula López Caballero y Ariadna Acevedo-Rodrigo, estas palabras son parte de los lenguajes culturales que usamos ordinariamente y también forman parte de los lenguajes institucionales y del estado que ordenan la vida social, pero sobre todo se trata de producciones políticas, es decir categorías de identificación que legitiman las diferencias sociales y al estado-nación. ${ }^{56}$ Tales categorías no surgen de manera inmediata, sino a través de procesos históricos que las estructuran y su definición no está determinada solamente por el estado o las instituciones, sino por las personas que las usan, por ello considero que el uso de la categoría lengua indígena es una arena de contiendas que se re-abren constantemente. Tal como el fragmento de Rosario Castellanos expresa, la lucha por la igualdad de las personas está mezclada con la perpetuación de las diferencias que se hace evidente en los discursos de la escritora. Aunque en los años setenta, se generó un movimiento reivindicativo de las lenguas, entonces ya categorizadas como indígenas, la lucha por su reconocimiento y valoración también está implicada en la ambigüedad del

56 Paula López Caballero y Ariadna Acevedo-Rodrigo «Introduction: Why Beyond Alterity?», en Beyond Alterity. Destabilizing the Indigenous Other in Mexico, eds. Paula López Caballero y Ariadna Acevedo-Rodrigo (Tucson: The University of Arizona Press, 2018), 10-11. 
término lengua indígena y en la desigualdad que este uso marca con respecto a las otras lenguas, es decir, las no indígenas.

Esta reflexión se orienta a repensar la educación del proyecto indigenista del INI de la década de 1950 como un proyecto institucional en el que no hubo un posicionamiento homogéneo durante todo el tiempo. La participación de distintas personas y sus propias contradicciones influyeron en la práctica indigenista de muchas maneras, sobre todo imprevisibles. Lo ocurrido con el títere Petul es muestra de lo inesperado. En las comunidades fue visto como un ser sabio, sobrenatural, casi sagrado y una autoridad merecedora de gran respeto. La gente de las comunidades invitaba a Petul como huésped, era invitado de honor en ceremonias, valoraban su consejo y pedían que volviera a presentarse. Los registros sobre Petul señalan que después de las presentaciones las gentes aceptaron realizar acciones de sanitarias en sus casas y comunidades, también aceptaron la apertura de caminos nuevos o se mostraban más accesibles para enviar a sus hijos a la escuela. ${ }^{57}$ Por esto los trabajos que han descrito las campañas del Teatro Petul lo han considerado un proyecto de gran éxito para la antropología y la política indigenista aplicada a la educación. ${ }^{58}$

A pesar de las aseveraciones acerca de los resultados exitosos del Teatro Petul, quedan ciertas dudas. La misma Rosario Castellanos dice: «Petul, de quien quisimos hacer un hombre de razón y se nos convirtió en un mito y en una fuerza sobrenatural». .59

\section{REFLEXIONES FINALES}

En este trabajo he mostrado que los cambios propiciados desde el indigenismo del CCI sí modificaron algunos usos del lenguaje, aunque los usos son ambivalentes. Las iniciativas del proyecto indigenista propiciaron el uso político del término «indígena» que, hasta la segunda década del siglo XXI, puede ser una manera de posicionarse como

\footnotetext{
57 Carlo Antonio Castro, «Educación indigenista: El Teatro Petul en los años cincuenta», Tramoya 67 (2001): 211-224. https://cdigital.uv.mx/bitstream/handle/123456789/4686/200167211.pdf;jsessionid=8927F166F390E53508C84256F0CD371F?sequence $=1$

58 Castro, 2001; Lewis, 2011.

59 Castellanos, «Teatro Petul», 31.
} 
alguien que no discrimina a ese otro o bien, cuando se usa como auto adscripción, marca la dignificación de los derechos ciudadanos del hablante. Desde los años setenta cuando comenzó a estudiarse la política indigenista de manera crítica, se advirtió el uso generalizado del término indígena tanto en los lenguajes políticos como en los académicos. Las demandas de los maestros autoproclamados indígenas influyeron en la creación de una dirección de educación indígena que prescribe de manera oficial la lectura y escritura en la lengua nativa de los niños al menos en los primeros años de educación básica.

En la formación del estado y de la sociedad mexicana la presencia de la población categorizada como indígena ha sido un dilema constante. Los textos de Rosario Castellanos son una evidencia de la pluralidad de voces sociales que resonaron tanto en su historia personal como en la política indigenista de mediados del siglo XX en México. En ellos se deja ver un discurso contradictorio, a veces racista y discriminador, mientras se expresan ideas acerca de la igualdad. Esos discursos son parte de los procesos de identificación del otro que están relacionados con la experiencia social acumulada personal y colectivamente. Los discursos y las prácticas de identificación y diferenciación son sitios de mediación complejos, interiorizados muchas veces y otras veces deliberados e intencionados como en los procesos educativos del INI que marcaban la diferencia para abogar por la igualdad.

En este trabajo he mostrado que entre las ideologías que subyacen a la defensa de la homogeneidad lingüística, están la creencia de que hace posible la comunicación y el mito de que genera igualdad. Pero los atributos negativos y las tensiones semánticas en la caracterización de la población categorizada como indígena se mantienen y se extienden a muchos otros aspectos, como la clasificación de sus lenguas.

El análisis que presento sobre los textos de Rosario Castellanos en donde alude a las lenguas tsotsil, tseltal y español busca evidenciar el uso contradictorio que ella hace de términos que pretenden ser neutrales semánticamente, así como las enunciaciones de los guiones en los que se expresan ideas contrarias y contradictorias con respecto a los indígenas y las lenguas que hablan. Mi propuesta es poner en perspectiva que esos usos no dependen solo de ella como persona, sino que concretan verbalmente luchas sociales, políticas, culturales y personales, dimensionadas 
históricamente con las luchas institucionales y las acciones políticas educativas para definir a la población considerada indígena, tomando en cuenta el factor de que son hablantes de lenguas distintas del español. Los conflictos lingüísticos implican conflictos sociales y los intentos por modificar la lengua, no tienen fines comunicativos sino fines sociales.

Con un alcance más general pretendo aportar a la discusión sobre la naturalidad con la que, en las esferas educativas institucionales se usa el término «lengua indígena» desde la última mitad del siglo XX a la fecha. Desde mi punto de vista, las contradicciones ideológicas y los desplazamientos semánticos que he mostrado en los textos de Rosario Castellanos se han asentado de manera contenciosa en el uso de este término. Las ideas sobre la igualdad de las lenguas parecen estar entrañadas en la fijación de la categoría de lengua indígena y aparentemente esta designación soluciona la conceptualización peyorativa. No obstante, el término de lenguas indígenas no conceptualiza a las lenguas debido a sus características estructurales, sino que se usa frecuentemente como explicativa de su naturaleza. Sin importar que tan distintas sean, todas esas lenguas se agrupan en la misma categoría porque se comparten las concepciones opuestas y contradictorias respecto de sus hablantes. Esta categoría en el lenguaje no alcanza para solucionar el conflicto social e incluir de manera realmente equitativa a los otros $\mathrm{y}$ a sus lenguas.

\section{Nota sobre la autora}

Susana Ayala Reyes es doctora en Ciencias en la especialidad de Investigaciones Educativas por el Centro de Investigaciones y Estudios Avanzados. Maestra en Ciencias del Lenguaje y licenciada en Etnología. Profesora de la Escuela Nacional de Antropología e Historia y la Universidad Nacional Autónoma de México. Sus líneas de investigación son antropología e historia de la educación indígena, lenguaje y comunicación en los procesos educativos escolarizados y no escolarizados, políticas educativas con relación a la lengua, análisis narrativo y del discurso, en ellas se vinculan la etnografía, la antropología lingüística y la historia. 


\section{REFERENCIAS}

Aguirre Beltrán, Gonzalo. Teoría y práctica de la educación indígena. Ciudad de México: Instituto Nacional Indigenista-Fondo de Cultura Económica, 1992. Aubry, Andrés. Les Tzotzil Par Eux-Mêmes. Récits et écrits de paysans indiens du Mexique. París: Éditions L'Harmattan, 1988.

Ayala Reyes, Susana. «Hands that Propose and Hands that Respond: Gestural Interaction in Petul-Xun Puppets Performances in Highland of Chiapas», Paedagogica Historica: International Journal of History of Education, 55 no. 2 (2019): 223-252. DOI: 10.1080/00309230.2018.1521447

Ayala Reyes, Susana. «Creatividad en los márgenes del estado: Apropiación de los títeres Petul-Xun en los Altos de Chiapas». PhD diss. Departamento de Investigaciones Educativas del Cinvestav, 2019.

Bakhtin, Mikhail M. «Epic and Novel». En The dialogic Imagination by M. M. Bakhtin, editado por Michael Holquist, 3-40. Austin: University of Texas Press, 1981).

Castellanos, Rosario «Teatro Petul», Revista de la Universidad de México XIX, no. 5, (1965): 30-31.

Cooper, Robert L. La planificación lingüística y el cambio social. Madrid: Cambridge University Press, 1997.

Gómez Gómez, Antonio. El Negro cimarrón. Ya'Yejal J-Ik'al. San Cristóbal de las Casas: UNAM, 2000.

Holland, Dorothy y Jean Lave. «History in Person: An Introduction». En History in Person. Enduring Struggles, Contentious Practices, Intimate Identities, editado por Dorothy Holland y Jean Lave, 3-33. Santa Fe: School of American Research Advanced Seminar, 2001.

Instituto Nacional Indigenista, Teatro Petul. México: Instituto Nacional Indigenista, 1956.

Instituto Nacional Indigenista, Teatro Petul 2. México: Instituto Nacional Indigenista, 1961.

Instituto Nacional Indigenista, Teatro Petul 3. México: Instituto Nacional Indigenista, 1962.

Jackson Albarrán, Elena. «Comino Vence Al Diablo and Other Terrifying Episodes: Teatro Guiñol's Itinerant Puppet Theater». En Seen and Heard in Mexico: Children and Revolutionary Cultural Nationalism, 175-212. Lincoln; London: University of Nebraska Press, 2014.

Jiménez Izquierdo, Juan. «Entrevista con Don Ferruco». Tramoya 10 (1978), 4953.

Lewis, Stephen E. Rethinking Mexican Indigenismo: The INI's Coordinating Center in Highland Chiapas and the Fate of a Utopian Project, 133-154; 155-174. Albuquerque: University of New Mexico Press, 2018. 
Lewis, Stephen E. «Modernizing Message, Mystical Messenger: The Teatro Petul in the Chiapas Highlans, 1954-1974». The Academy of American Franciscan History, 67, no. 3, (2011): 375-397. http://muse.jhu.edu/journals/tam/ summary/v067/67.3.lewis.html

López Caballero, Paula. «Las políticas indigenistas y la 'fábrica' de su sujeto de intervención en la creación del primer Centro Coordinador del Instituto Nacional Indigenista (1948-1952)». En Nación y alteridad. Mestizos, indígenas y extranjeros en el proceso de formación nacional, coordinado por Daniela Gleizer y Paula López Caballero, 69-108. México D.F.: Universidad Autónoma Metropolitana/Ediciones EyC, 2015.

López Caballero, Paula y Ariadna Acevedo-Rodrigo, eds. «Introduction: Why Beyond Alterity?» En Beyond Alterity. Destabilizing the Indigenous Other in Mexico, 3-28. Tucson: The University of Arizona Press, 2018.

López García, María. "Aprendan el "vosotros" para hablar con los españoles en su idioma: La identificación lingüística en Argentina como tensión entre el orgullo y la minusvaloración», Historia y Memoria de la Educación, 2 (2015): 97-124. DOI:10.5944/hme.2.2015.14234

Medina Hernández, Andrés. «Estudio preliminar. La trama, los hilos y los nudos de un proyecto de investigación. La Universidad de Chicago en los Altos de Chiapas». En R. Guber, La articulación etnográfica, 11-34. Buenos Aires, Argentina: Editorial Biblos, 2013.

Navarrete Cáceres, Carlos. Rosario Castellanos, su presencia en la antropología mexicana. México, D.F.: Instituto de Investigaciones Antropológicas de la Universidad Nacional Autónoma de México-Programa de Investigaciones Multidisciplinarios sobre Mesoamérica y el Sureste, 2007.

Pitarch Ramón, Pedro. «Un lugar difícil: Estereotipos étnicos y juego de poder en Los Altos de Chiapas». En Chiapas: Los rumbos de otra historia, editado por Juan Pedro Viqueira y Mario Humberto Ruz, 237-250. México, D.F.: Universidad Nacional Autónoma de México, Centro de Investigaciones y Estudios Superiores en Antropología Social, Centro de Estudios Mexicanos y Centroamericanos, Universidad de Guadalajara, 1995.

Sommers, Joseph. "Forma e ideología en "Oficio de tinieblas” de Rosario Castellanos", Revista de Crítica Literaria Latinoamericana, 2, no. 7/8 (1978): 7391. DOI: $10.2307 / 4529870$

Viqueira, Juan Pedro. "Las causas de una rebelión india: Chiapas, 1712». En Chiapas los rumbos de otra historia, editado por Juan Pedro Viqueira y Mario Humberto Ruz. D.F., 103-143. México: Universidad Nacional Autónoma de México, Centro de Investigaciones y Estudios Superiores en Antropología Social, Centro de Estudios Mexicanos y Centroamericanos, Universidad de Guadalajara, 1995. 
Voloshinov, V. N. y Bajtín, M. La construcción de la enunciación. Buenos Aires: Editorial Almagesto, 1930/1978.

Zabaltza Pérez-Nievas, Xabier. Historia de las lenguas y los nacionalismos. Barcelona: Gedisa, 2006.

\section{Archivos}

AHCCITT. Archivo Histórico Agustín Romano Delgado del Centro Coordinador Indigenista Tzeltal- Tzotzil. San Cristóbal de las Casas, Chiapas. Sección Educación, Sección Dirección, Sección Educación, Sección Lingüística. 